\title{
Calculation of the Effective Delayed Neutron Fraction by Deterministic and Monte Carlo Methods
}

\author{
M. Carta, ${ }^{1}$ S. Dulla, ${ }^{2}$ V. Peluso, ${ }^{3}$ P. Ravetto, ${ }^{2}$ and G. Bianchini ${ }^{1}$ \\ ${ }^{1}$ ENEA, C.R. Casaccia, Via Anguillarese 301, 00123 Roma, Italy \\ ${ }^{2}$ Dipartimento di Energetica, Politecnico di Torino, Corso Duca degli Abruzzi 24, 10129 Torino, Italy \\ ${ }^{3}$ ENEA, C.R.E. E. Clementel, Via Martiri di Monte Sole 4, 40129 Bologna, Italy \\ Correspondence should be addressed to S.Dulla, sandra.dulla@polito.it
}

Received 7 March 2011; Accepted 5 May 2011

Academic Editor: Antonio Alvim

Copyright (c) 2011 M. Carta et al. This is an open access article distributed under the Creative Commons Attribution License, which permits unrestricted use, distribution, and reproduction in any medium, provided the original work is properly cited.

The studies on Accelerator-Driven Systems (ADSs) have renewed the interest in the theoretical and computational evaluation of the main integral parameters characterizing subcritical systems (e.g., reactivity, effective delayed neutron fraction $\beta_{\text {eff }}$, and mean prompt neutron generation time). In particular, some kinetic parameters, as the effective delayed neutron fraction, are evaluated in Monte Carlo codes by formulations which do not require the calculation of the adjoint flux. This paper is focused on a theoretical and computational analysis about how the different $\beta_{\text {eff }}$ definitions are connected and which are the approximations inherent to the Monte Carlo definition with respect to the standard definition involving weighted integrals. By means of a refined transport computational analysis carried out in a coherent and consistent way, that is, using the same deterministic code and neutron data library for the $\beta_{\text {eff }}$ evaluation in different ways, the theoretical analysis is numerically confirmed. Both theoretical and numerical results confirm the effectiveness of the Monte Carlo $\beta_{\text {eff }}$ evaluation, at least in cases where spectral differences between total and prompt fluxes are negligible with respect to the value of the functionals entering the classical $\beta_{\mathrm{eff}}$ formulation.

\section{Introduction}

The studies on Accelerator-Driven Systems (ADSs) have renewed the interest in the theoretical and computational evaluation of the main integral parameters characterizing subcritical systems (e.g., reactivity, effective delayed neutron fraction, and mean neutron generation time [1]). In particular, the extensive use of Monte Carlo codes for the analysis of the ADS neutronic behaviour is challenging the deterministic codes, used in the past for the analysis of critical systems, for what concerns the capability to reproduce Monte Carlo results, usually taken as reference [24]. Nevertheless, some particular parameters, as the effective delayed neutron fraction, are evaluated in Monte Carlo codes by formulations which do not require the calculation of the adjoint flux. The assessment of the various formulations of the effective delayed neutron fraction is crucial for the system evaluation, since it plays an important role in determining its dynamic characteristics [5]. This paper is focused on a theoretical and computational analysis on the connections among the different $\beta_{\text {eff }}$ definitions, with special attention to the approximations inherent to the Monte Carlo definition with respect to the standard definition involving weighted integrals. Theoretical results show how the Monte Carlo formulation of $\beta_{\text {eff }}$ may be related to the classical definition, interpreting the classical one through a reactivity evaluation based on an "improved" first-order approach of perturbation theory. The computational analysis is carried out in a coherent and consistent way, using the same deterministic code and neutron data library for the $\beta_{\text {eff }}$ evaluations. A simplified system allows to investigate the features of the various procedures and to use the results to obtain a physical insight. The GUINEVERE system is then selected as a relevant test case for ADS technology. The GUINEVERE experience $[6,7]$, mainly devoted to the issues concerning online reactivity monitoring in ADS, is analysed by using a modified layout of the VENUS critical facility located at the $\mathrm{SCK} \bullet \mathrm{CEN}$ site in Mol, Belgium, coupling the subcritical core facility to a deuteron accelerator delivering $14 \mathrm{MeV}$ neutrons by deuterium-tritium reactions, by a continuous or pulsed 
beam. For the GUINEVERE experience the ERANOS system [8] and neutron data library JEFF 3.1 [9] have been used to perform transport calculations with 49 energy groups for a cylindrical schematization of the GUINEVERE start-up (at critical) configuration. The analysis of the results allows to draw some conclusions on the merits and the limits of the various formulations.

\section{Theoretical Analysis}

2.1. The General Case. Let us take as reference system the following eigenvalue problem:

$$
\mathbf{L} \varphi=\omega \mathbf{F} \varphi,
$$

where $\mathbf{L}$ is the loss operator, $\mathbf{F}$ the fission operator, and $\omega=1 / k$, inverse of the classical multiplication eigenvalue. Then, a perturbation $\delta \mathbf{F}$ is introduced in the system. As a consequence, the eigenvalue problem for the perturbed system is

$$
\mathbf{L} \varphi^{*}=[\omega+\delta \omega][\mathbf{F}+\delta \mathbf{F}] \varphi^{*},
$$

with $\varphi^{*}$ as the perturbed flux. Following the exact approach of the Perturbation Theory (PT) [10], that is, considering the adjoint problem corresponding to the reference system

$$
\mathbf{L}^{+} \varphi^{+}=\omega \mathbf{F}^{+} \varphi^{+},
$$

one obtains (the symbol $\langle\cdots\rangle$ denotes integration over the full phase space, while, in the following, $\langle\cdots\rangle_{x}$ is denoting integration only over the specified variable $x$ ):

$$
\frac{\delta \omega}{\omega+\delta \omega}=-\frac{\left\langle\varphi^{+} \delta \mathbf{F} \varphi^{*}\right\rangle}{\left\langle\varphi^{+} \mathbf{F} \varphi^{*}\right\rangle}
$$

or equivalently

$$
\frac{\delta \omega}{\omega}=-\frac{\left\langle\varphi^{+} \delta \mathbf{F} \varphi^{*}\right\rangle}{\left\langle\varphi^{+}(\mathbf{F}+\delta \mathbf{F}) \varphi^{*}\right\rangle} .
$$

If $\omega+\delta \omega=1 / k^{*}$, we can write (4) in terms of the $k$ eigenvalue:

$$
1-\frac{k^{*}}{k}=-\frac{\left\langle\varphi^{+} \delta \mathbf{F} \varphi^{*}\right\rangle}{\left\langle\varphi^{+} \mathbf{F} \varphi^{*}\right\rangle} .
$$

If we write (2) as

$$
\mathbf{L}[\varphi+\delta \varphi]=[\omega+\delta \omega][\mathbf{F}+\delta \mathbf{F}][\varphi+\delta \varphi]
$$

and follow the first-order $\mathrm{PT}$ approach, while retaining the second-order term $\delta \omega \delta \mathbf{F} \varphi$, we obtain the "improved" firstorder formulation

$$
\frac{\delta \omega}{\omega+\delta \omega} \cong-\frac{\left\langle\varphi^{+} \delta \mathbf{F} \varphi\right\rangle}{\left\langle\varphi^{+} \mathbf{F} \varphi\right\rangle}
$$

or, in terms of the $k$ eigenvalue,

$$
1-\frac{k^{*}}{k} \cong-\frac{\left\langle\varphi^{+} \delta \mathbf{F} \varphi\right\rangle}{\left\langle\varphi^{+} \mathbf{F} \varphi\right\rangle} .
$$

2.2. The "Prompt" Perturbation. If the perturbation is assumed to be equal to the delayed neutrons fission operator $\mathbf{F}_{d}$, hence $\delta \mathbf{F}=-\mathbf{F}_{d}$, the perturbed system given in (2) can be written as

$$
\mathbf{L} \varphi_{p}=\omega_{p}\left[\mathbf{F}-\mathbf{F}_{\mathbf{d}}\right] \varphi_{p},
$$

with the perturbed flux given by the prompt flux $\varphi_{p}$ and $\omega_{p}=1 / k_{p}$. Then (6) becomes

$$
1-\frac{k_{p}}{k}=\frac{\left\langle\varphi^{+} \mathbf{F}_{\mathbf{d}} \varphi_{p}\right\rangle}{\left\langle\varphi^{+} \mathbf{F} \varphi_{p}\right\rangle}
$$

and (9) takes the following form:

$$
1-\frac{k_{p}}{k} \cong \frac{\left\langle\varphi^{+} \mathbf{F}_{\mathbf{d}} \varphi\right\rangle}{\left\langle\varphi^{+} \mathbf{F} \varphi\right\rangle}=\beta_{\text {eff. }}
$$

Thus, the effective delayed neutron fraction $\beta_{\text {eff }}$ is an "improved" PT first-order formulation of the relationship $1-\left(k_{p} / k\right)$, widely used in Monte Carlo codes as $\beta_{\text {eff }}$ estimator. The pure PT first-order formulation provides:

$$
\frac{\delta \omega}{\omega}=\frac{k}{k_{p}}-1 \cong-\frac{\left\langle\varphi^{+} \delta \mathbf{F} \varphi\right\rangle}{\left\langle\varphi^{+} \mathbf{F} \varphi\right\rangle}=\frac{\left\langle\varphi^{+} \mathbf{F}_{\mathbf{d}} \varphi\right\rangle}{\left\langle\varphi^{+} \mathbf{F} \varphi\right\rangle}=\beta_{\text {eff. }}
$$

To pass from (11) to (12), it is sufficient to replace $\varphi_{p}$ by $\varphi$.

\section{Standard and Monte Carlo $\beta_{\text {eff }}$ Formulations}

The effectiveness of delayed neutrons is normally obtained by one of the following two relationships:

$$
\begin{gathered}
\beta_{\mathrm{eff}}=\sum_{m} \sum_{i} \bar{v}_{d, i}^{(m)} \frac{\left\langle\left\langle\Phi^{+}(\mathbf{r}, E) \chi_{d, i}^{(m)}(E)\left\langle\sum_{f}^{(m)}\left(\mathbf{r}, E^{\prime}\right) \Phi\left(\mathbf{r}, E^{\prime}\right)\right\rangle_{E^{\prime}}\right\rangle_{E}\right\rangle_{\mathbf{r}}\left\langle\left\langle\Phi^{+}(\mathbf{r}, E) \chi^{(m)}(E)\left\langle v^{(m)}\left(E^{\prime}\right) \Sigma_{f}^{(m)}\left(\mathbf{r}, E^{\prime}\right) \Phi\left(\mathbf{r}, E^{\prime}\right)\right\rangle_{E^{\prime}}\right\rangle_{E}\right\rangle_{\mathbf{r}}}{\sum_{\mathbf{r}}}, \\
\tilde{\beta}_{\mathrm{eff}}=\sum_{m} \sum_{i} \beta_{i}^{(m)} \frac{\left\langle\left\langle\Phi^{+}(\mathbf{r}, E) \chi_{d, i}^{(m)}(E)\left\langle\nu^{(m)}\left(E^{\prime}\right) \Sigma_{f}^{(m)}\left(\mathbf{r}, E^{\prime}\right) \Phi\left(\mathbf{r}, E^{\prime}\right)\right\rangle_{E^{\prime}}\right\rangle_{E}\right\rangle_{\mathbf{r}}}{\sum_{m}\left\langle\left\langle\Phi^{+}(\mathbf{r}, E) \chi^{(m)}(E)\left\langle\gamma^{(m)}\left(E^{\prime}\right) \Sigma_{f}^{(m)}\left(\mathbf{r}, E^{\prime}\right) \Phi\left(\mathbf{r}, E^{\prime}\right)\right\rangle_{E^{\prime}}\right\rangle_{E}\right\rangle_{\mathbf{r}}},
\end{gathered}
$$


where $m$ is the fissile isotope index, $i$ the delayed neutron family index, $\chi_{d, i}^{(m)}$ the delayed neutron spectrum for fissile isotope $m$ and delayed neutron family $i$ (basic data), $\chi^{(m)}$ the total neutron spectrum for fissile isotope $m, \bar{v}_{d, i}^{(m)}$ the average value of delayed neutrons emitted from fissile isotope $m$ and delayed neutron family $i$, for a given incident neutron spectrum (from basic data), $v^{(m)}$ the total neutrons emitted from fissile isotope $m$, and $\Sigma_{f}^{(m)}$ the macroscopic fission cross section for fissile isotope $m$.

Furthermore,

$$
\beta_{i}^{(m)}=\frac{\bar{\nu}_{d, i}^{(m)}}{\bar{\nu}_{\psi}^{(m)}}
$$

with $\bar{\nu}_{\psi}^{(m)}$ being the average value of the number of total neutrons emitted from the fissile isotope $m$ for a given incident neutron spectrum $\psi(\psi$ has to be calculated by a cell or system neutronic calculation). In (14) and (15) the direct flux and the adjoint flux are solutions for the following eigenvalue equations, respectively:

$$
\begin{aligned}
\boldsymbol{\Omega} & \nabla \varphi(\mathbf{r}, \boldsymbol{\Omega}, E)+\Sigma_{t}(\mathbf{r}, E) \varphi(\mathbf{r}, \boldsymbol{\Omega}, E) \\
= & \frac{1}{k} \sum_{m} \chi^{(m)}(E)<v^{(m)}\left(E^{\prime}\right) \Sigma_{f}^{(m)}\left(\mathbf{r}, E^{\prime}\right) \Phi\left(\mathbf{r}, E^{\prime}\right)>_{E^{\prime}} \\
& +<\Sigma_{s}\left(\mathbf{r}, E^{\prime} \longrightarrow E\right) \Phi\left(\mathbf{r}, E^{\prime}\right)>_{E^{\prime}}
\end{aligned}
$$

$$
\begin{aligned}
-\boldsymbol{\Omega} & \cdot \nabla \varphi^{+}(\mathbf{r}, \boldsymbol{\Omega}, E)+\Sigma_{t}(\mathbf{r}, E) \varphi^{+}(\mathbf{r}, \boldsymbol{\Omega}, E) \\
= & \frac{1}{k} \sum_{m} v^{(m)}(E) \Sigma_{f}^{(m)}(\mathbf{r}, E)\left\langle\chi^{(m)}\left(E^{\prime}\right) \Phi^{+}\left(\mathbf{r}, E^{\prime}\right)\right\rangle_{E^{\prime}} \\
& +\left\langle\Sigma_{s}\left(\mathbf{r}, E \longrightarrow E^{\prime}\right) \Phi^{+}\left(\mathbf{r}, E^{\prime}\right)\right\rangle_{E^{\prime}} .
\end{aligned}
$$

Current Monte Carlo (MC) calculations approximate $\beta_{\text {eff }}$ by the following formula:

$$
\beta_{\mathrm{eff}}^{\mathrm{MC}}=1-\frac{k_{p}}{k}
$$

where $k_{p}$ is the multiplication eigenvalue of the equation

$$
\begin{aligned}
\boldsymbol{\Omega} \cdot & \nabla \varphi_{p}(\mathbf{r}, \boldsymbol{\Omega}, E)+\Sigma_{t}(\mathbf{r}, E) \varphi_{p}(\mathbf{r}, \boldsymbol{\Omega}, E) \\
= & \frac{1}{k_{p}} \sum_{m}\left[\chi^{(m)}(E)-\sum_{i} \chi_{d, i}^{(m)}(E) \beta_{i}^{(m)}(\mathbf{r})\right] \\
& \times\left\langle\nu^{(m)}\left(E^{\prime}\right) \Sigma_{f}^{(m)}\left(\mathbf{r}, E^{\prime}\right) \Phi_{p}\left(\mathbf{r}, E^{\prime}\right)\right\rangle_{E^{\prime}} \\
& +\left\langle\Sigma_{s}\left(\mathbf{r}, E^{\prime} \longrightarrow E\right) \Phi_{p}\left(\mathbf{r}, E^{\prime}\right)\right\rangle_{E^{\prime}}
\end{aligned}
$$

Equation (20) is written in a general form, taking into account the spatial dependence of the delayed emission fraction $\beta$ on position $\mathbf{r}$. In this case $\beta_{i}^{(m)}(\mathbf{r})$ is given by

$$
\beta_{i}^{(m)}(\mathbf{r})=\frac{\bar{v}_{d, i}^{(m)}}{\left[\left\langle\nu^{(m)}\left(E^{\prime}\right) \Sigma_{f}^{(m)}\left(\mathbf{r}, E^{\prime}\right) \Phi_{p}\left(\mathbf{r}, E^{\prime}\right)\right\rangle_{E^{\prime}} /\left\langle\Sigma_{f}^{(m)}\left(\mathbf{r}, E^{\prime}\right) \Phi_{p}\left(\mathbf{r}, E^{\prime}\right)\right\rangle_{E^{\prime}}\right]} .
$$

If we consider the system governed by (20) as a perturbation of the system governed by (17), we obtain as exact perturbation (11), that is,

$$
1-\frac{k_{p}}{k}=\sum_{m} \sum_{i} \frac{\left\langle\left\langle\Phi^{+}(\mathbf{r}, E) \chi_{d, i}^{(m)}(E) \beta_{i}^{(m)}(\mathbf{r})\left\langle\nu^{(m)}\left(E^{\prime}\right) \Sigma_{f}^{(m)}\left(\mathbf{r}, E^{\prime}\right) \Phi_{p}\left(\mathbf{r}, E^{\prime}\right)\right\rangle_{E^{\prime}}\right\rangle_{E}\right\rangle_{\mathbf{r}}}{\sum_{m}\left\langle\left\langle\Phi^{+}(\mathbf{r}, E) \chi^{(m)}(E)\left\langle\nu^{(m)}\left(E^{\prime}\right) \Sigma_{f}^{(m)}\left(\mathbf{r}, E^{\prime}\right) \Phi_{p}\left(\mathbf{r}, E^{\prime}\right)\right\rangle_{E^{\prime}}\right\rangle_{E}\right\rangle_{\mathbf{r}}},
$$

where $\varphi^{+}$is the eigenfunction of (18) and $\varphi_{p}$ the eigenfunction of (20). When comparing (22) with (15), it can be noticed that, besides the replacement of $\varphi$ by $\varphi_{p}$, the differences lie in the presence of the terms $\beta_{i}^{(m)}(\mathbf{r})$ (under integration) replacing the terms $\beta_{i}^{(m)}$. If we assume in both (20) and (22) the following approximation:

$$
\begin{aligned}
\beta_{i}^{(m)}(\mathbf{r}) & \approx \beta_{i}^{(m)} \\
& =\frac{\bar{\nu}_{d, i}^{(m)}}{\bar{\nu}_{\psi}^{(m)}},
\end{aligned}
$$

equation (20) takes the following form:

$$
\begin{aligned}
\boldsymbol{\Omega} \cdot & \nabla \varphi_{p}(\mathbf{r}, \boldsymbol{\Omega}, E)+\sum_{t}(\mathbf{r}, E) \varphi_{p}(\mathbf{r}, \boldsymbol{\Omega}, E) \\
= & \frac{1}{\widetilde{k}_{p}} \sum_{m}\left[\chi^{(m)}(E)-\sum_{i} \chi_{d, i}^{(m)}(E) \beta_{i}^{(m)}\right] \\
& \times\left\langle\nu^{(m)}\left(E^{\prime}\right) \Sigma_{f}^{(m)}\left(\mathbf{r}, E^{\prime}\right) \Phi_{p}\left(\mathbf{r}, E^{\prime}\right)\right\rangle_{E^{\prime}} \\
& +\left\langle\Sigma_{s}\left(\mathbf{r}, E^{\prime} \longrightarrow E\right) \Phi_{p}\left(\mathbf{r}, E^{\prime}\right)\right\rangle_{E^{\prime}}
\end{aligned}
$$


and (22) becomes

$$
\begin{aligned}
& \widetilde{\beta}_{\mathrm{eff}}^{\mathrm{MC}}=1-\frac{\tilde{k}_{p}}{k} \\
& =\sum_{m} \sum_{i} \beta_{i}^{(m)} \\
& \times \frac{\left\langle\left\langle\Phi^{+}(\mathbf{r}, E) \chi_{d, i}^{(m)}(E)\left\langle v^{(m)}\left(E^{\prime}\right) \Sigma_{f}^{(m)}\left(\mathbf{r}, E^{\prime}\right) \Phi_{p}\left(\mathbf{r}, E^{\prime}\right)\right\rangle_{E^{\prime}}\right\rangle_{E}\right\rangle_{\mathbf{r}}}{\sum_{m}\left\langle\left\langle\Phi^{+}(\mathbf{r}, E) \chi^{(m)}(E)\left\langle\nu^{(m)}\left(E^{\prime}\right) \Sigma_{f}^{(m)}\left(\mathbf{r}, E^{\prime}\right) \Phi_{p}\left(\mathbf{r}, E^{\prime}\right)\right\rangle_{E^{\prime}}\right\rangle_{E}\right\rangle_{\mathbf{r}}} .
\end{aligned}
$$

As shown in the previous section, the classical $\beta_{\text {eff }}$ definition given in (15) is an "improved" PT first-order approximation of the relationship $1-\left(k_{p} / k\right)$ given in (25). Along the point of view of the perturbation approach, leading to a relationship among system integral properties like the eigenvalues and the delayed neutron effectiveness, (24) and (25) are intimately coupled, in the sense that the coherent prompt flux $\varphi_{p}$ to be used in the $\beta_{\text {eff }}$ definition given by (25) is the eigenfunction of (24). We recall that (24) is an approximation of (20), owing to the assumption given in (23). If we want to remove this assumption, we have to make explicit the $\beta(\mathbf{r})$ definition given in (21) and insert it into (20), thus obtaining

$$
\begin{aligned}
& \boldsymbol{\Omega} \cdot \nabla \varphi_{p}(\mathbf{r}, \boldsymbol{\Omega}, E)+\Sigma_{t}(\mathbf{r}, E) \varphi_{p}(\mathbf{r}, \boldsymbol{\Omega}, E) \\
&=\frac{1}{k_{p}} \sum_{m}\left[\chi^{(m)}(E)\left\langle\nu^{(m)}\left(E^{\prime}\right) \Sigma_{f}^{(m)}\left(\mathbf{r}, E^{\prime}\right) \Phi_{p}\left(\mathbf{r}, E^{\prime}\right)\right\rangle_{E^{\prime}}\right. \\
&\left.\quad-\sum_{i} \chi_{d, i}^{(m)}(E) \bar{\nu}_{d, i}^{(m)}\left\langle\Sigma_{f}^{(m)}\left(\mathbf{r}, E^{\prime}\right) \Phi_{p}\left(\mathbf{r}, E^{\prime}\right)\right\rangle_{E^{\prime}}\right] \\
&+\left\langle\Sigma_{s}\left(\mathbf{r}, E^{\prime} \longrightarrow E\right) \Phi_{p}\left(\mathbf{r}, E^{\prime}\right)\right\rangle_{E^{\prime}} .
\end{aligned}
$$

Following the same perturbation approach, that is, perturbed system given by (26) and unperturbed system given by (17), we find

$$
\beta_{\mathrm{eff}}^{\mathrm{MC}}=1-\frac{k_{p}}{k}=\sum_{m} \sum_{i} \bar{\nu}_{d, i}^{(m)} \frac{\left\langle\left\langle\Phi^{+}(\mathbf{r}, E) \chi_{d, i}^{(m)}(E)\left\langle\Sigma_{f}^{(m)}\left(\mathbf{r}, E^{\prime}\right) \Phi_{p}\left(\mathbf{r}, E^{\prime}\right)\right\rangle_{E^{\prime}}\right\rangle_{E}\right\rangle_{\mathbf{r}}}{\sum_{m}\left\langle\left\langle\Phi^{+}(\mathbf{r}, E) \chi^{(m)}(E)\left\langle\nu^{(m)}\left(E^{\prime}\right) \Sigma_{f}^{(m)}\left(\mathbf{r}, E^{\prime}\right) \Phi_{p}\left(\mathbf{r}, E^{\prime}\right)\right\rangle_{E^{\prime}}\right\rangle_{E}\right\rangle_{\mathbf{r}}} .
$$

Analogously, the classical $\beta_{\mathrm{eff}}$ definition given in (14) is an "improved" PT first-order approximation of the relationship $1-\left(k_{p} / k\right)$ given in (27). Also in this case (26) and (27) are intimately coupled, in the sense that the coherent prompt flux $\varphi_{p}$ to be used in the $\beta_{\text {eff }}$ definition given by (27) is the eigenfunction of (26). The previous elaboration can be summarized in the following way:

(a) as introduced in the previous section, the classical $\beta_{\text {eff }}$ definitions, like those given in (14) and (15), which do not require any prompt flux calculation, are "improved" PT first-order approximations of the relationship $1-\left(k_{p} / k\right)$ used by Monte Carlo calculations;

(b) the relationship $1-\left(k_{p} / k\right)$ provides a $\beta_{\text {eff }}$ estimate coherent with the assumptions made when performing the prompt flux calculation, that is, (24) provides the estimate given by (25), and (26) provides the estimate given by (27).

\section{ERANOS Formalism and Calculation Set up}

The following $\beta_{\text {eff }}$ formulation is adopted in ERANOS [11] (given in the energy multigroup scheme, with $g$ as energy group index):

$$
\beta_{\mathrm{eff}}=\sum_{m} \sum_{i} \bar{\nu}_{d, i}^{(m)} \frac{\left\langle\sum_{g} \Phi_{g}^{+}(\mathbf{r}) \bar{\chi}_{d, i, g} \sum_{g^{\prime}} \Sigma_{f, g^{\prime}}^{(m)}(\mathbf{r}) \Phi_{g^{\prime}}(\mathbf{r})\right\rangle_{\mathbf{r}}}{\left\langle\sum_{g} \Phi_{g}^{+}(\mathbf{r}) \bar{\chi}_{g} \sum_{m} \sum_{g^{\prime}} v_{g^{\prime}}^{(m)} \Sigma_{f, g^{\prime}}^{(m)}(\mathbf{r}) \Phi_{g^{\prime}}(\mathbf{r})\right\rangle_{\mathbf{r}}}
$$

where $\bar{\chi}_{d, i, g}$ is a unique delayed neutron spectrum (derived from basic data) for each fissile isotope $m$, delayed neutron family $i$, and

$$
\bar{\chi}_{g}=\frac{\sum_{m} \chi_{g}^{(m)} \sum_{g^{\prime}} v_{g^{\prime}}^{(m)} \Sigma_{f, g^{\prime}}^{(m)} \psi_{g^{\prime}}}{\sum_{m} \sum_{g^{\prime}} v_{g^{\prime}}^{(m)} \Sigma_{f, g^{\prime}}^{(m)} \psi_{g^{\prime}}} .
$$

The quantity $\bar{\chi}_{g}$ is a unique fission spectrum, used by ERANOS for spatial calculations, for all the fissile isotopes. The weighting flux $\psi_{g}$ is obtained by the fuel cell cross section calculation. In (28) the direct flux and the adjoint flux are solutions for the following eigenvalue equations, respectively:

$$
\begin{aligned}
\boldsymbol{\Omega} & \cdot \nabla \varphi_{g}(\mathbf{r}, \boldsymbol{\Omega})+\sum_{t, g}(\mathbf{r}) \varphi_{g}(\mathbf{r}, \boldsymbol{\Omega}) \\
= & \frac{1}{k} \bar{\chi}_{g} \sum_{m} \sum_{g^{\prime}} v_{g^{\prime}}^{(m)} \Sigma_{f, g^{\prime}}^{(m)}(\mathbf{r}) \Phi_{g^{\prime}}(\mathbf{r})+\sum_{g^{\prime}} \sum_{s, g^{\prime} \rightarrow g}(\mathbf{r}) \Phi_{g^{\prime}}(\mathbf{r}) \\
- & \boldsymbol{\Omega} \cdot \nabla \varphi_{g}^{+}(\mathbf{r}, \boldsymbol{\Omega})+\sum_{t, g}(\mathbf{r}) \varphi_{g}^{+}(\mathbf{r}, \boldsymbol{\Omega}) \\
& =\frac{1}{k} \sum_{m} v_{g}^{(m)} \sum_{f, g}^{(m)}(\mathbf{r}) \sum_{g^{\prime}} \bar{\chi}_{g^{\prime}} \Phi_{g^{\prime}}^{+}(\mathbf{r})+\sum_{g^{\prime}} \Sigma_{s, g \rightarrow g^{\prime}}(\mathbf{r}) \Phi_{g^{\prime}}^{+}(\mathbf{r})
\end{aligned}
$$

It can be seen that, besides the approximation on the delayed neutron spectrum and on the total fission spectrum, (28) is the energy-multigroup version of (14). Considering 


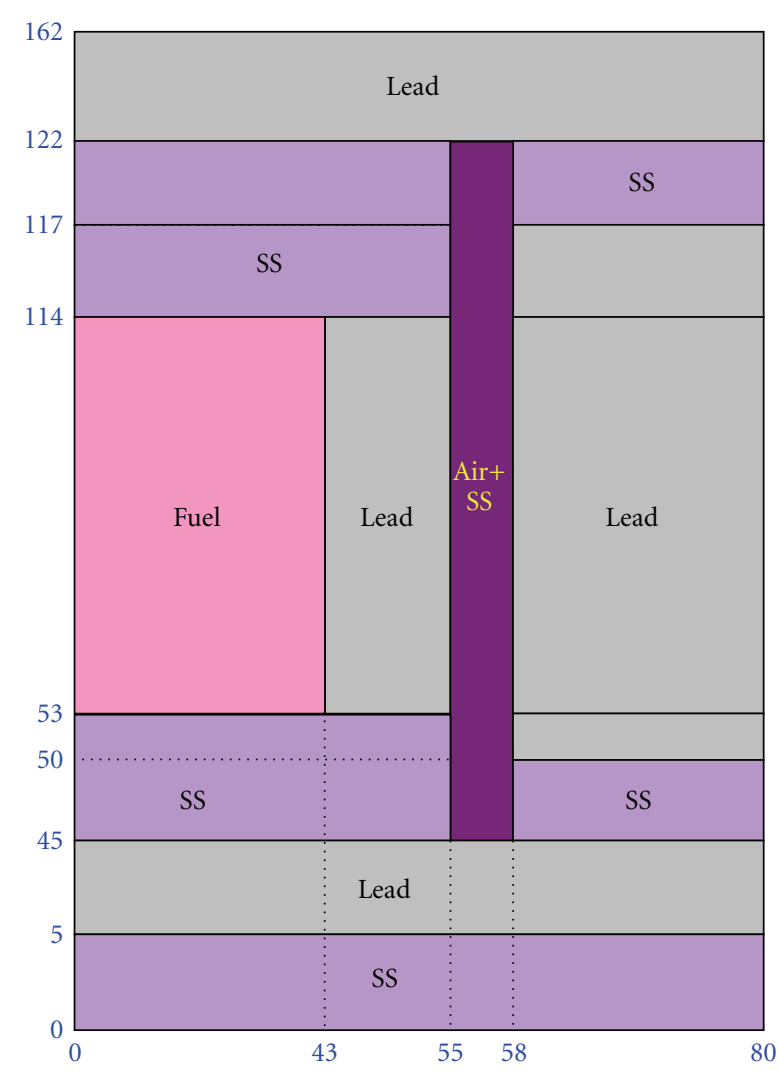

Figure 1: Simplified RZ model of the GUINEVERE start-up (at critical) configuration. Dimensions, not in scale, are given in $\mathrm{cm}$.

as perturbed state with respect to the reference state in (30) the solution for the following equation:

$$
\begin{aligned}
\boldsymbol{\Omega} & \cdot \nabla \varphi_{p, g}(\mathbf{r}, \boldsymbol{\Omega})+\Sigma_{t, g}(\mathbf{r}) \varphi_{p, g}(\mathbf{r}, \boldsymbol{\Omega}) \\
= & \frac{1}{k_{p}} \sum_{m} \sum_{g^{\prime}}\left[\bar{\chi}_{g} \nu_{g^{\prime}}^{(m)}-\sum_{i} \bar{\chi}_{d, i, g} \bar{\nu}_{d, i}^{(m)}\right] \sum_{f, g^{\prime}}^{(m)}(\mathbf{r}) \Phi_{p, g^{\prime}}(\mathbf{r}) \\
& +\sum_{g^{\prime}} \Sigma_{s, g^{\prime} \rightarrow g}(\mathbf{r}) \Phi_{p, g^{\prime}}(\mathbf{r}),
\end{aligned}
$$

we obtain as exact perturbation

$$
\begin{aligned}
\beta_{\mathrm{eff}}^{\mathrm{MC}}= & 1-\frac{k_{p}}{k} \\
= & \sum_{m} \sum_{i} \bar{\nu}_{d, i}^{(m)} \\
& \times \frac{\left\langle\sum_{g} \Phi_{g}^{+}(\mathbf{r}) \bar{\chi}_{d, i, g} \sum_{g^{\prime}} \Sigma_{f, g^{\prime}}^{(m)}(\mathbf{r}) \Phi_{p, g^{\prime}}(\mathbf{r})\right\rangle_{\mathbf{r}}}{\left\langle\sum_{g} \Phi_{g}^{+}(\mathbf{r}) \bar{\chi}_{g} \sum_{m} \sum_{g^{\prime}} v_{g^{\prime}}^{(m)} \Sigma_{f, g^{\prime}}^{(m)}(\mathbf{r}) \Phi_{p, g^{\prime}}(\mathbf{r})\right\rangle_{\mathbf{r}}} .
\end{aligned}
$$

On the basis of the theory outlined in the previous section, (28) is the "improved" PT first-order approximation of (33). Unfortunately, ERANOS cannot calculate the solution for (32) and consequently (33) cannot be applied because, as

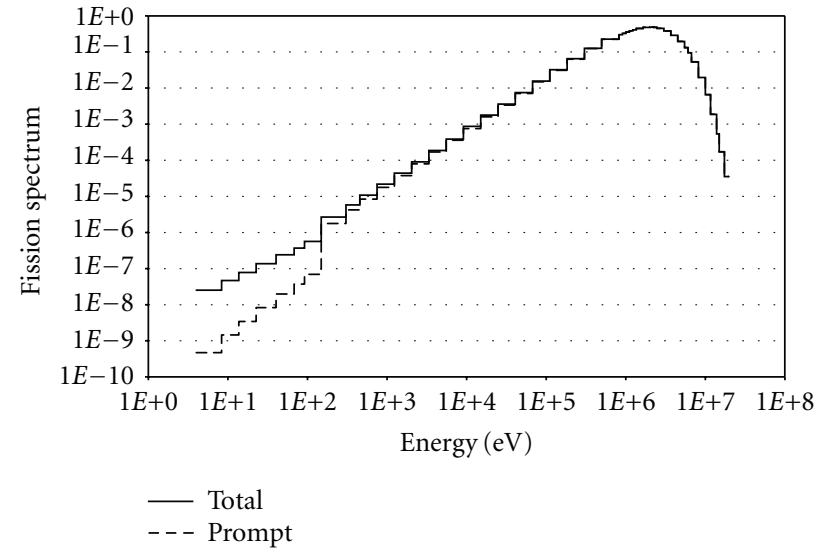

Figure 2: Total fission spectrum $\bar{\chi}_{g}$ (37) and prompt fission spectrum $\bar{\chi}_{p, g}(38)$.
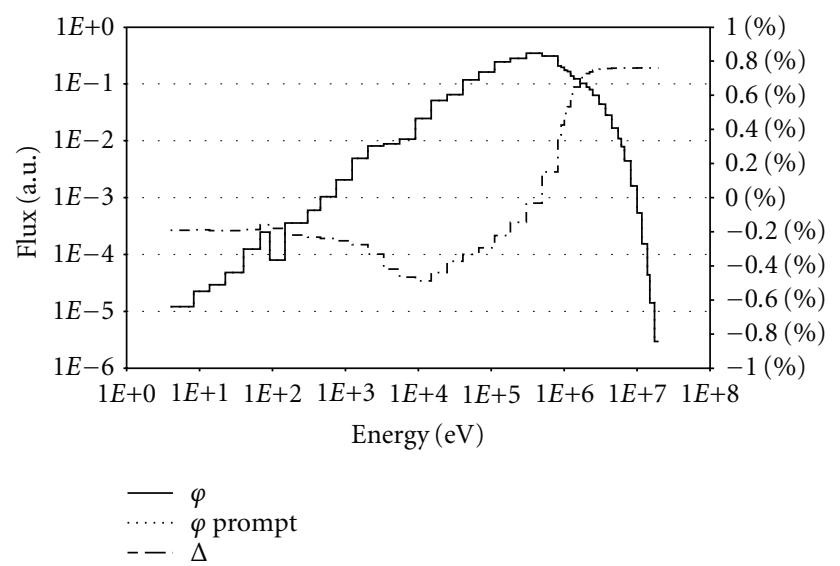

Figure 3: Total and prompt fluxes, together with spectral differences $\Delta=\left(\varphi_{p, g}-\varphi_{g}\right) / \varphi_{g}$. $\left(\varphi_{p, g}\right.$ from case (f) and $\varphi_{g}$ from case (d) in Table 2, spectral differences on right axis).

mentioned before, it uses a unique fission spectrum for all the fissile isotopes (and in (32) both $\bar{\chi}_{g}$ and the various $\bar{\chi}_{d, i, g}$ appear). In addition, to use (for our scopes) the $\beta_{\text {eff }}$ modules built-in in ERANOS we need a further assumption, that is, we have to evaluate

$$
\bar{\nu}^{(m)}=\frac{\sum_{g^{\prime}} v_{g^{\prime}}^{(m)} \sum_{f, g^{\prime}}^{(m)} \psi_{g^{\prime}}}{\sum_{g^{\prime}} \sum_{f, g^{\prime}}^{(m)} \psi_{g^{\prime}}},
$$

with $\psi_{g}$ as weighting flux obtained by the cell cross-section calculation. Thus, our new reference state is given by the following equation:

$$
\begin{aligned}
\boldsymbol{\Omega} & \nabla \varphi_{g}(\mathbf{r}, \boldsymbol{\Omega})+\sum_{t, g}(\mathbf{r}) \varphi_{g}(\mathbf{r}, \boldsymbol{\Omega}) \\
= & \frac{1}{k} \bar{\chi}_{g} \sum_{m} \bar{\nu}^{(m)} \sum_{g^{\prime}} \sum_{f, g^{\prime}}^{(m)}(\mathbf{r}) \Phi_{g^{\prime}}(\mathbf{r}) \\
& +\sum_{g^{\prime}} \sum_{s, g^{\prime} \rightarrow g}(\mathbf{r}) \Phi_{g^{\prime}}(\mathbf{r}),
\end{aligned}
$$


TABLE 1: Delayed neutron data $\bar{v}_{d, i}^{(m)}$ (from [12]).

\begin{tabular}{lccccccccc}
\hline Family & 1 & 2 & 3 & 4 & 5 & 6 & 7 & 8 & Sum \\
\hline $\mathrm{U}_{235}$ & 0.00055 & 0.00245 & 0.00162 & 0.00326 & 0.00509 & 0.00152 & 0.00142 & 0.00039 & 0.01630 \\
$\mathrm{U}_{238}$ & 0.00039 & 0.00484 & 0.00174 & 0.00637 & 0.01367 & 0.00921 & 0.00595 & 0.00433 & 0.04650 \\
\hline
\end{tabular}

TABLE 2: Synthesis of the results.

\begin{tabular}{|c|c|c|c|c|c|}
\hline Case & Equation & $k$ & $k_{p}$ & $\begin{array}{c}\beta_{\mathrm{eff}} \\
(\mathrm{pcm})\end{array}$ & $\begin{array}{c}1-k_{p} / k \\
(\mathrm{pcm})\end{array}$ \\
\hline (a) & (28) for $\beta_{\mathrm{eff}}$ & & & 723 & \\
\hline (b) & (31) for $\varphi$ & 1.01955 & & & \\
\hline (c) & (31) for $\varphi^{+}$ & 1.01955 & & & \\
\hline (d) & (35) for $\varphi$ & 1.02149 & & & \\
\hline (e) & (36) for $\varphi^{+}$ & 1.02149 & & & \\
\hline (f) & (39) for $\varphi_{p}$ & & 1.01402 & & \\
\hline (g) & (41) for $1-k_{p} / k$ & & & & 732 \\
\hline (h) & (41) for $\beta_{\text {eff }}$ (using $\varphi_{p}$ ) & & & 732 & \\
\hline (i) & $(42)$ for $\beta_{\text {eff }}(u \operatorname{sing} \varphi)$ & & & 731 & \\
\hline
\end{tabular}

with the associated adjoint

$$
\begin{aligned}
- & \boldsymbol{\Omega} \cdot \nabla \varphi_{g}^{+}(\mathbf{r}, \boldsymbol{\Omega})+\sum_{t, g}(\mathbf{r}) \varphi_{g}^{+}(\mathbf{r}, \boldsymbol{\Omega}) \\
& =\frac{1}{k} \sum_{m} \bar{\nu}^{(m)} \sum_{f, g}^{(m)}(\mathbf{r}) \sum_{g^{\prime}} \bar{\chi}_{g^{\prime}} \Phi_{g^{\prime}}^{+}(\mathbf{r})+\sum_{g^{\prime}} \sum_{s, g \rightarrow g^{\prime}}(\mathbf{r}) \Phi_{g^{\prime}}^{+}(\mathbf{r}),
\end{aligned}
$$

where (of course, because of (34), (37) provides the same result as (29)):

$$
\bar{\chi}_{g}=\frac{\sum_{m} \chi_{g}^{(m)} \bar{\nu}^{(m)} \sum_{g^{\prime}} \Sigma_{f, g^{\prime}}^{(m)} \psi_{g^{\prime}}}{\sum_{m} \bar{\nu}^{(m)} \sum_{g^{\prime}} \Sigma_{f, g^{\prime}}^{(m)} \psi_{g^{\prime}}}
$$

Then, setting

$$
\bar{\chi}_{p, g}=\frac{\sum_{m}\left[\bar{\chi}_{g} \bar{\nu}^{(m)}-\sum_{i} \bar{\chi}_{d, i, g} \bar{\nu}_{d, i}^{(m)}\right] \sum_{g^{\prime}} \sum_{f, g^{\prime}}^{(m)} \psi_{g^{\prime}}}{\sum_{m}\left[\bar{\nu}^{(m)}-\sum_{i} \bar{\nu}_{d, i}^{(m)}\right] \sum_{g^{\prime}} \sum_{f, g^{\prime}}^{(m)} \psi_{g^{\prime}}},
$$

Equation (32) becomes

$$
\begin{aligned}
\boldsymbol{\Omega} \cdot & \nabla \varphi_{p, g}(\mathbf{r}, \boldsymbol{\Omega})+\sum_{t, g}(\mathbf{r}) \varphi_{p, g}(\mathbf{r}, \boldsymbol{\Omega}) \\
= & \frac{1}{k_{p}} \bar{\chi}_{p, g} \sum_{m}\left[\bar{\nu}^{(m)}-\sum_{i} \bar{\nu}_{d, i}^{(m)}\right] \sum_{g^{\prime}} \sum_{f, g^{\prime}}^{(m)}(\mathbf{r}) \Phi_{p, g^{\prime}}(\mathbf{r}) \\
& +\sum_{g^{\prime}} \sum_{s, g^{\prime} \rightarrow g}(\mathbf{r}) \Phi_{p, g^{\prime}}(\mathbf{r}) .
\end{aligned}
$$

On the basis of (16), (39) is equivalent to:

$$
\begin{aligned}
\boldsymbol{\Omega} \cdot & \nabla \varphi_{p, g}(\mathbf{r}, \boldsymbol{\Omega})+\sum_{t, g}(\mathbf{r}) \varphi_{p, g}(\mathbf{r}, \boldsymbol{\Omega}) \\
= & \frac{1}{k_{p}} \bar{\chi}_{p, g} \sum_{m}\left[1-\sum_{i} \beta_{i}^{(m)}\right] \bar{\nu}^{(m)} \sum_{g^{\prime}} \sum_{f, g^{\prime}}^{(m)}(\mathbf{r}) \Phi_{p, g^{\prime}}(\mathbf{r}) \\
& +\sum_{g^{\prime}} \sum_{s, g^{\prime} \rightarrow g}(\mathbf{r}) \Phi_{p, g^{\prime}}(\mathbf{r})
\end{aligned}
$$

Considering now (39) as the perturbed state with respect to the reference state in (35), taking into account (38), we obtain

$$
\begin{aligned}
\beta_{\mathrm{eff}}^{\mathrm{MC}}= & 1-\frac{k_{p}}{k} \\
= & \sum_{m} \sum_{i} \bar{\nu}_{d, i}^{(m)} \\
& \times \frac{\left\langle\sum_{g} \Phi_{g}^{+}(\mathbf{r}) \bar{\chi}_{d, i, g} \sum_{g^{\prime}} \sum_{f, g^{\prime}}^{(m)}(\mathbf{r}) \Phi_{p, g^{\prime}}(\mathbf{r})\right\rangle_{\mathbf{r}}}{\left\langle\sum_{g} \Phi_{g}^{+}(\mathbf{r}) \bar{\chi}_{g} \sum_{m} \bar{\nu}^{(m)} \sum_{g^{\prime}} \Sigma_{f, g^{\prime}}^{(m)}(\mathbf{r}) \Phi_{p, g^{\prime}}(\mathbf{r})\right\rangle_{\mathbf{r}}} .
\end{aligned}
$$

The corresponding “improved” PT first-order approximation of (41) is

$$
\beta_{\mathrm{eff}}=\sum_{m} \sum_{i} \bar{\nu}_{d, i}^{(m)} \frac{\left\langle\sum_{g} \Phi_{g}^{+}(\mathbf{r}) \bar{\chi}_{d, i, g} \sum_{g^{\prime}} \sum_{f, g^{\prime}}^{(m)}(\mathbf{r}) \Phi_{g^{\prime}}(\mathbf{r})\right\rangle_{\mathbf{r}}}{\left\langle\sum_{g} \Phi_{g}^{+}(\mathbf{r}) \bar{\chi}_{g} \sum_{m} \bar{\nu}^{(m)} \sum_{g^{\prime}} \Sigma_{f, g^{\prime}}^{(m)}(\mathbf{r}) \Phi_{g^{\prime}}(\mathbf{r})\right\rangle_{\mathbf{r}}} .
$$

and Equation (39), both sides of (41) and (42) can be calculated by ERANOS.

\section{ERANOS Results}

The GUINEVERE system, shown in Figure 1, is selected to perform the numerical analysis. The fuel fissile zone is made of Uranium $30 \%$ (weight) enriched in $\mathrm{U}_{235}$. The fuel matrix has the following volumetric fractions: Uranium 17\%, Stainless Steel 16\%, Lead 60\% and Air 7\%. The zone Air + SS Sheet has the following volumetric fractions: Stainless Steel $93 \%$ and Air 7\%. The RZ system is a simplified schematization of the GUINEVERE start-up (at critical) configuration (after start-up the central zone is replaced by the beam tube of the deuteron accelerator delivering, in continuous or pulsed mode, $14 \mathrm{MeV}$ neutrons by deuteriumtritium reactions).

ERANOS transport calculations are performed with 49 energy groups (see the appendix), $\mathrm{P}_{0}$ transport approximation, and angular quadrature $\mathrm{S}_{4}$ (module BISTRO [8]). The neutron data library JEFF 3.1 [9] is used for the calculations. In Table 1 the delayed neutron data $\bar{v}_{d, i}^{(m)}$ [12] used for the analysis are shown. Delayed neutron spectra $\bar{\chi}_{d, i, g}$ are the ones built-in in ERANOS and taken from [12].

The following $\bar{\nu}^{(m)}$ values were obtained by (28): $\bar{\nu}^{(235)}=2.49$ and $\bar{\nu}^{(238)}=2.75$. This high $\bar{\nu}^{(238)}$ value is due to the fact that about $61 \%$ of $\mathrm{U}^{(238)}$ total fissions occur in the energy range $2 \div 8 \mathrm{MeV}$, where $v^{(238)}$ values span from 2.67 to 3.48 (only about $5 \%$ of $\mathrm{U}^{(238)}$ total fissions occur above $2 \mathrm{MeV}$ ). In Figure 2 a comparison between the 
TABLE 3: The 49 energy group scheme.

\begin{tabular}{|c|c|c|c|c|c|c|c|}
\hline $\begin{array}{l}\text { Upper energy } \\
(\mathrm{eV})\end{array}$ & $\begin{array}{c}\text { Group } \\
\#\end{array}$ & $\begin{array}{c}\text { Upper energy } \\
(\mathrm{eV})\end{array}$ & $\begin{array}{c}\text { Group } \\
\#\end{array}$ & $\begin{array}{c}\text { Upper energy } \\
(\mathrm{eV})\end{array}$ & $\begin{array}{c}\text { Group } \\
\#\end{array}$ & $\begin{array}{c}\text { Upper energy } \\
(\mathrm{eV})\end{array}$ & $\begin{array}{c}\text { Group } \\
\#\end{array}$ \\
\hline $1.96 E+07$ & 1 & $2.470 E+06$ & 14 & $1.11 E+05$ & 27 & $1.49 E+02$ & 40 \\
\hline $1.73 E+07$ & 2 & $2.23 E+06$ & 15 & $6.74 E+04$ & 28 & $9.17 E+01$ & 41 \\
\hline $1.49 E+07$ & 3 & $2.02 E+06$ & 16 & $4.09 E+04$ & 29 & $6.79 E+01$ & 42 \\
\hline $1.38 E+07$ & 4 & $1.65 E+06$ & 17 & $2.48 E+04$ & 30 & $4.02 E+01$ & 43 \\
\hline $1.16 E+07$ & 5 & $1.35 E+06$ & 18 & $1.50 E+04$ & 31 & $2.26 E+01$ & 44 \\
\hline $1.00 E+07$ & 6 & $1.22 E+06$ & 19 & $9.12 E+03$ & 32 & $1.37 E+01$ & 45 \\
\hline $8.19 E+06$ & 7 & $1.11 E+06$ & 20 & $5.53 E+03$ & 33 & $8.32 E+00$ & 46 \\
\hline $6.70 E+06$ & 8 & $1.00+06$ & 21 & $3.35 E+03$ & 34 & $4.000 E+00$ & 47 \\
\hline $6.07 E+06$ & 9 & $9.07 E+05$ & 22 & $2.03 E+03$ & 35 & $5.40 E-01$ & 48 \\
\hline $5.49 E+06$ & 10 & $8.21 E+05$ & 23 & $1.23 E+03$ & 36 & $1.00 E-01$ & 49 \\
\hline $4.49 E+06$ & 11 & $4.98 E+05$ & 24 & $7.49 E+02$ & 37 & & \\
\hline $3.68 E+06$ & 12 & $3.02 E+05$ & 25 & $4.54 E+02$ & 38 & & \\
\hline $3.04 E+06$ & 13 & $1.83 E+05$ & 26 & $3.04 E+02$ & 39 & & \\
\hline
\end{tabular}

total fission spectrum $\bar{\chi}_{g}$ obtained by (37) and the prompt fission spectrum $\bar{\chi}_{p, g}$ obtained by (38) is shown. In Table 2 a synthesis of the results is reported.

Case (a) is the classical $\beta_{\text {eff }}$ formulation adopted in ERANOS, with $\varphi$ and $\varphi^{+}$from cases (b) and (c), respectively. Cases (d) and (e) differ from (b) and (c) for the presence of $\bar{v}^{(m)}$ in the fission production terms, and this approximation provides a difference of $+186 \mathrm{pcm}$ with respect to the standard treatment of the fission production terms. The prompt case (f), again with the presence of $\bar{\nu}^{(m)}$ in the fission production terms, provides a difference of $-721 \mathrm{pcm}\left(\sim \beta_{\text {eff }}\right)$ with respect to cases (d) and (e).

Case $(\mathrm{g})$, representing the approach used by Monte Carlo calculations, provides exactly the same value as in case (h), that is, the last term in (41). This numerical result confirms the theoretical analysis described above, linking the Monte Carlo formulation to the PT approach. Finally, case (i) shows that the replacement of the prompt flux in case (h) by the total flux does not affect appreciably the $\beta_{\text {eff }}$ value (at least for the fast system considered).

Actually, the differences between $\varphi$ and $\varphi_{p}$, from cases (d) and (f) in Table 2, respectively, are not remarkable, as it can be seen from Figure 3 (where $\varphi$ and $\varphi_{p}$ are undistinguishable). $\varphi$ and $\varphi_{p}$ in Figure 3 are spatial average values over the core volume, same area under $\varphi$ and $\varphi_{p}$ curves.

\section{Conclusions}

When evaluating $\beta_{\text {eff }}$ by the relationship $1-\left(k_{p} / k\right)$, the quality of the obtained results depends on the quality of the description of the delayed neutron emissions assumed in the $k_{p}$ prompt calculation. It could seem obvious, but if we look at the standard route used in ERANOS (and in general in deterministic codes) to calculate $\beta_{\text {eff }}$, by a formulation which does not require any prompt flux calculation, we realize that great detail is given to the characteristics of the delayed neutron emission through the $\beta_{\text {eff }}$ formulation, and without the need to transpose such a detail into a full system prompt neutron calculation. For example, in the ERANOS case, it is not possible to set up a prompt calculation having the same quality of the delayed neutron emission description available in the $\beta_{\text {eff }}$ formulation. And, in fact, in the present case we are obliged to lower the level of the information about the delayed neutron emission in the $\beta_{\text {eff }}$ definition in order to run the corresponding prompt calculation.

Following the perturbation theory approach, a rigorous relationship may be established between the $\beta_{\text {eff }}$ evaluation by the formula $1-\left(k_{p} / k\right)$, used in Monte Carlo codes, and the corresponding $\beta_{\text {eff }}$ calculation by the classical formulation involving direct and adjoint fluxes, and it has been shown how the classical formulation can be considered an "improved" PT first-order formulation of the formula 1$\left(k_{p} / k\right)$. By means of a transport computational analysis using a 49 energy group structure and carried out in a coherent and consistent way, that is, using the same deterministic code ERANOS and neutron data library JEFF 3.1 for the $\beta_{\text {eff }}$ evaluation in different ways, the theoretical analysis is numerically confirmed.

Both theoretical and numerical results confirm the effectiveness of the $\beta_{\text {eff }}$ evaluation by the relationship $1-\left(k_{p} / k\right)$, at least in cases where spectral differences between total and prompt fluxes are negligible with respect to the value of the functionals entering the classical $\beta_{\text {eff }}$ formulation. For other material configurations more investigations are needed to obtain a detailed quantification of the effects involved.

\section{Appendix}

The 49 energy group structure, presented in Table 3, has been adopted in order to capture the various spectral phenomena associated to the source-driven subcritical assembly. Different built-in energy group grids are available into ERANOS. In this work the base library at 1968 energy groups, together with two derived energy grids at 172 and 33 energy groups, has been used to produce the cross sections. 
The 49 energy group structure has been obtained using the standard 33 energy group below $0.82 \mathrm{MeV}$ and the finest 172 energy group above this energy in order to better take into account the D-T neutron external source foreseen for the GUINEVERE subcritical configurations (neutron energy at about $14 \mathrm{MeV})$.

\section{References}

[1] S. Dulla, P. Picca, D. Tomatis, P. Ravetto, and M. Carta, "Integral parameters in source-driven systems," Progress in Nuclear Energy, vol. 53, no. 1, pp. 32-40, 2011.

[2] R. K. Meulekamp and S. C. van der Marck, "Calculating the effective delayed neutron fraction with Monte Carlo," Nuclear Science and Engineering, vol. 152, no. 2, pp. 142-148, 2006.

[3] B. Verboomen, W. Haeck, and P. Baeten, "Monte Carlo calculation of the effective neutron generation time," Annals of Nuclear Energy, vol. 33, no. 10, pp. 911-916, 2006.

[4] Y. Nagaya and T. Mori, "Calculation of effective delayed neutron fraction with Monte Carlo perturbation techniques," Annals of Nuclear Energy, vol. 38, no. 2-3, pp. 254-260, 2011.

[5] Y. Nagaya, G. Chiba, T. Moria, D. Irwanto, and K. Nakajima, "Comparison of Monte Carlo calculation methods for effective delayed neutron fraction," Annals of Nuclear Energy, vol. 37, no. 10, pp. 1308-1315, 2010.

[6] P. Baeten, H. Ait Abderrahim, T. Aoust et al., "The GUINEVERE project at the VENUS facility," in Proceedings of the International Conference on the Physics of Reactors (PHYSOR '08), vol. 4, pp. 2867-2873, Interlaken, Switzerland, September 2008.

[7] http://www.sckcen.be/en/Our-Research/Research-projects/ EU-projects-FP6-FP7/GUINEVERE.

[8] G. Rimpault et al., "The ERANOS code and data system for fast reactor neutronic analyses," in Proceedings of the International Conference on the Physics of Reactors, (PHYSOR '02), Seoul, Korea, October 2002.

[9] A. Santamarina, D. Bernard, P. Blaise et al., "The JEFF-3.1.1 Nuclear Data Library—Report 22," NEA 6807, Nuclear Energy Agency, 2009.

[10] A. Gandini, "A generalized perturbation method for bi-linear functionals of the real and adjoint neutron fluxes," Journal of Nuclear Energy, vol. 21, no. 10, pp. 755-765, 1967.

[11] V. Zammit and E. Fort, "Constantes de Neutrons Retardés pour le calcul du $\beta_{\text {eff }}$ avec ERANOS," CEA Technical Note NT-SPRC-LEPh-97-230, 1997.

[12] A. D'Angelo and J. L. Rowlands, "Conclusions concerning the delayed neutron data for the major actinides," Progress in Nuclear Energy, vol. 41, no. 1-4, pp. 391-412, 2002. 

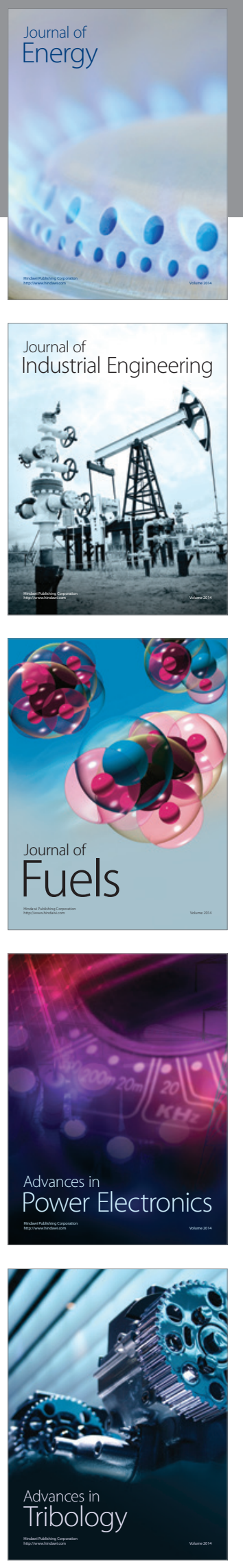
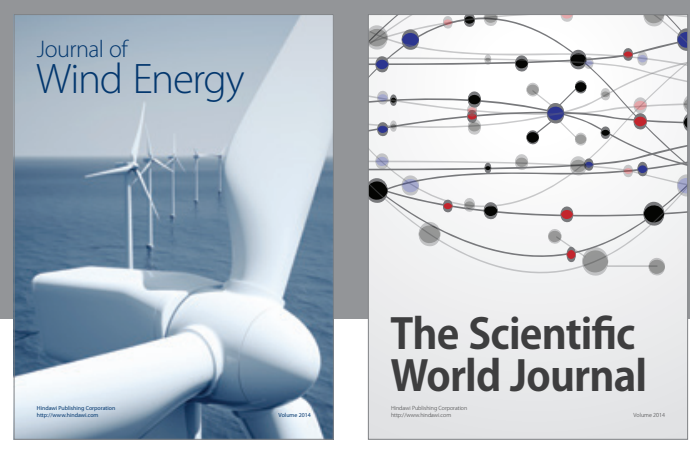

The Scientific World Journal

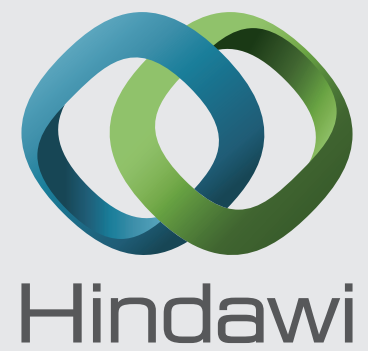

Submit your manuscripts at http://www.hindawi.com
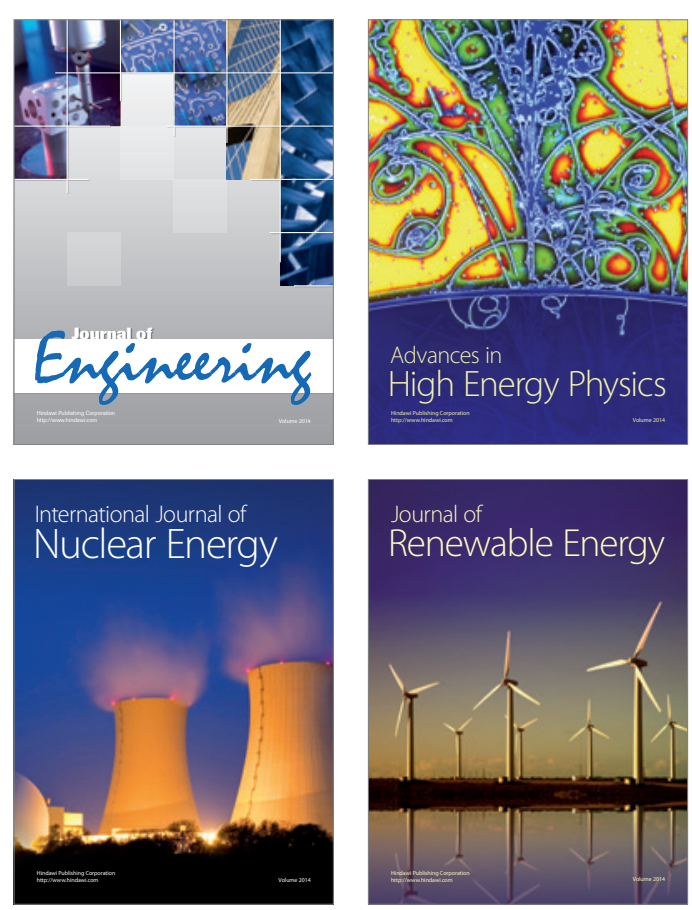

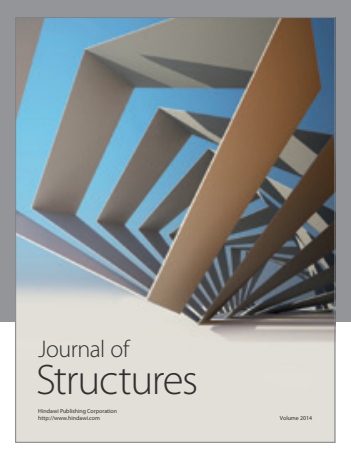

Rotating
Mechinery
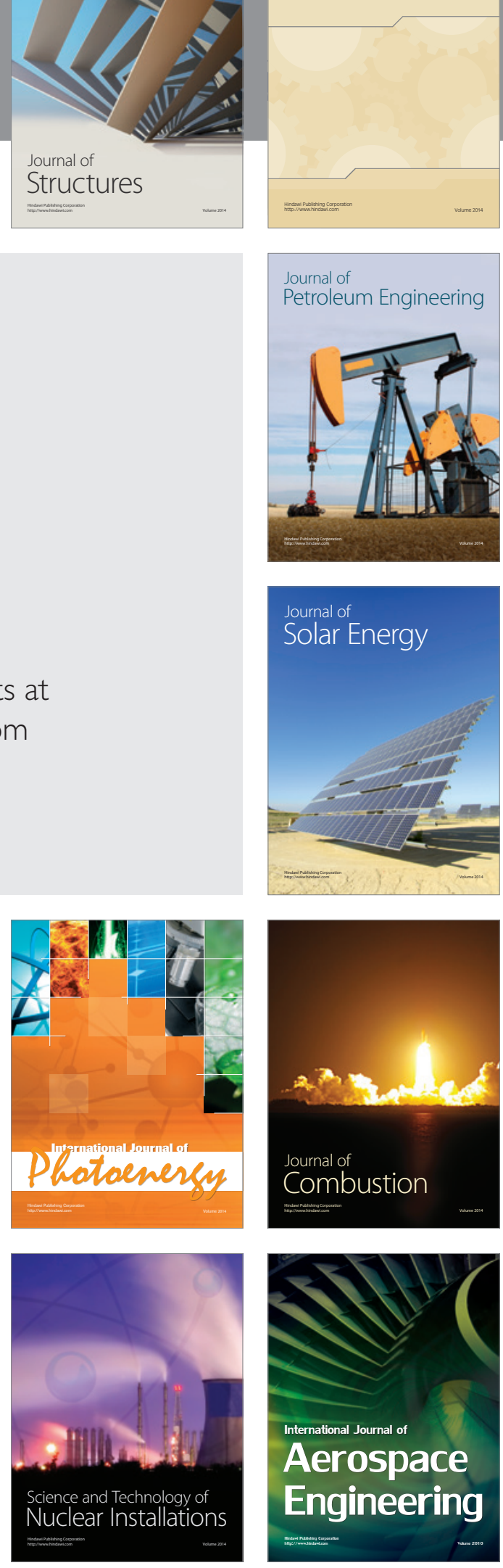